\title{
Staphylococcal intramammary infection dynamics and the relationship with milk quality parameters in dairy goats over the dry period
}

\author{
Véronique Bernier Gosselin, ${ }^{1 *}$ Simon Dufour, ${ }^{2,3}$ Michael J. Calcutt, ${ }^{4}$ Pamela R. F. Adkins, ${ }^{1}$ \\ and John R. Middleton ${ }^{1} \dagger$ \\ ${ }^{1}$ Department of Veterinary Medicine and Surgery, College of Veterinary Medicine, University of Missouri, 900 East Campus Dr., Columbia 65211 \\ ${ }^{2}$ Department of Pathology and Microbiology, Faculty of Veterinary Medicine, Université de Montréal, 3200 Sicotte, Saint-Hyacinthe, QC, Canada, \\ J2S $2 \mathrm{M} 2$ \\ ${ }^{3}$ Canadian Bovine Mastitis and Milk Quality Research Network, 3200 Sicotte, Saint-Hyacinthe, QC, Canada, J2S 2M2 \\ ${ }^{4}$ Department of Veterinary Pathobiology, College of Veterinary Medicine, University of Missouri, 900 East Campus Dr., Columbia 65211
}

\section{ABSTRACT}

The objectives of this study were (1) to report the rates of new intramammary infection (IMI) and spontaneous IMI cure over the dry period in 3 dairy goat herds; (2) to evaluate the factors predicting infection dynamics over the dry period; and (3) to define milk quality parameter thresholds that predict infection dynamics over the dry period. Two consecutive udder-half milk samples were collected 10 to $14 \mathrm{~d}$ apart before dry-off from 288 goats in 3 herds, and 2 consecutive udder-half samples were collected 7 to $14 \mathrm{~d}$ apart in the following lactation, with the first sample being collected $\leq 10 \mathrm{~d}$ in milk, from 200 of the same goats. In 2 of the herds, udder-half milk samples were also collected at the same time points $(\mathrm{n}=312$ halves; 157 goats) for measurement of milk quality parameters. Standard aerobic culture of milk samples was performed for the detection of mastitis pathogens. To rule out the presence of Mycoplasma spp. IMI, milk samples were also cultured on modified Hayflick medium. NonMycoplasma isolates were speciated using MALDI-TOF mass spectrometry. Staphylococcal isolates, when not identified by MALDI-TOF, were speciated using partial gene sequence analysis of $r p o B$ or $t u f$. When $>1$ sample from an udder half yielded the same species, available isolates from the first and last positive samples for that species were strain-typed using pulsed-field gel electrophoresis. Incidence of new IMI and cure rate were computed. Generalized linear mixed regression models were built to evaluate the associations between new IMI and pre-dry somatic cell score (SCS), between IMI persistence and half-level SCS, and between IMI persistence and pre-dry IMI species. Thresholds for

Received November 21, 2018.

Accepted January 22, 2019

*Current address: Department of Clinical Sciences, Faculty of Veterinary Medicine, Université de Montréal, 3200 Sicotte, SaintHyacinthe, QC, Canada, J2S 2M2.

†Corresponding author: middletonjr@missouri.edu pre-dry SCS and lactose concentration were computed to predict IMI persistence. Overall, $12.6 \%(48 / 380)$ of halves had a persistent IMI. Cumulative incidence of new IMI over the dry period was $13.2 \%$, and cure rate was $52.0 \%$. Pre-dry SCS was not associated with odds of new IMI or IMI persistence. Pre-dry IMI species was not associated with odds of persistence. Lactose concentration was not associated with odds of persistence. Regardless of culture data, the optimal pre-dry SCS threshold to detect IMI that would persist into the next lactation was 8.7, with sensitivity and specificity of 50 and $73.8 \%$, respectively. Further studies on the effect of control measures on species-specific incidence and cure rates during the dry period are warranted.

Key words: caprine, mastitis, dry period, persistence, staphylococci

\section{INTRODUCTION}

Staphylococci are the most common cause of subclinical IMI in dairy goats, with a half-level prevalence ranging from 20 to $40 \%$ (Contreras et al., 1995; White and Hinckley, 1999). In goats $\leq 4$ DIM in 2 studies, the prevalence of NAS IMI was 8 to $12.2 \%$ (McDougall et al., 2010, 2014). Many staphylococcal species are reported to cause persistent IMI during lactation (Contreras et al., 1997), and prevalence of IMI increases with stage of lactation (Moroni et al., 2005b). At dry-off, half-level staphylococcal IMI prevalences ranging from 4.7 to $25.5 \%$ were reported (Leitner et al., 2007; Bastan et al., 2015). Persistence of staphylococcal IMI from one lactation to the next was also reported (Poutrel, 1984) and could partly explain the presence of IMI at the onset of lactation. In cows, intramammary antimicrobials are often administered on the day of drying off to treat existing IMI and prevent new IMI during the dry period, and some studies have reported the use of dry-off treatment in dairy goats (Poutrel et al., 1997; Bastan et al., 2015). However, the dynamics of staphylococcal IMI around the dry period in goats are poorly 
documented. Reported new IMI rates during the dry period range from 4.2 to $7.5 \%$ (Fox et al., 1992; Leitner et al., 2007). In one study, the spontaneous cure rate of NAS IMI during the dry period was $19.7 \%$ (Poutrel et al., 1997). However, comparisons between studies are made difficult by the various time intervals between pre-dry sampling and dry-off or between kidding and post-kidding sampling, various dry-period durations, and use of dry-off antibiotic therapy.

Persistence of IMI has been defined as repeated isolation from an udder half of the same species over time (Contreras et al., 1997), but this definition may lead to false conclusions. Using pulsed-field gel electrophoresis (PFGE) strain-typing, our research group observed that during lactation, some halves infected with a given NAS species eliminate the initial IMI and become infected with a different strain of the same species (Bernier Gosselin et al., 2019). The same phenomenon is likely to occur during the dry period but has not been investigated.

Although late lactation is associated with an increase in SCC in uninfected cows and goats (Leitner et al., 2012), the magnitude of this increase in goats is such that SCC may not be helpful for differentiating infected and uninfected udder halves in late lactation (Moroni et al., 2005b). There is a paucity of data on characteristics, such as SCC at dry-off or species of staphylococci causing IMI, that could be used to differentiate IMI that persist from IMI that are spontaneously cured during the dry period and, therefore, may or may not warrant intramammary antibiotic treatment in the dry period. The objectives of this study were (1) to report the species-specific staphylococcal IMI dynamics over the dry period, including the new IMI and spontaneous IMI cure; (2) to evaluate the factors predicting infection dynamics over the dry period; and (3) to define SCC or lactose concentration thresholds that predict infection dynamics over the dry period.

\section{MATERIALS AND METHODS}

\section{Herds}

The study protocol and all procedures were approved by the University of Missouri Animal Care and Use Committee. The study design was an observational longitudinal study. The goats enrolled in this study resided on 3 commercial dairy farms. Herd 1 was in year-round production and goats were enrolled that were due to be dried off within a few weeks after study initiation. Herds 2 and 3 were in seasonal production; all goats were dried off at the same time. Hence, all goats in herds 2 and 3 that were still producing milk at the time of study initiation (within a few weeks of dry-off) were included in the study. No enrolled goats in any of the 3 herds received any antimicrobial treatment during the study. Demographic and management data for each herd are shown in Table 1. During lactation, all goats were machine-milked in a parlor twice daily. In herds 1 and 2 , kids were separated from the dams, and the does were reintroduced into the milking herd $1 \mathrm{~d}$ (herd 1) or 5 milkings (herd 2) after kidding. In herd 3, the kids were nursing on the dams, and the does were milked by hand once a day for the first $2 \mathrm{wk}$ in lactation; then, the kids were separated and the does were machine-milked twice a day. Data for parity, DIM, and dry-off and kidding dates were retrieved from each herd's records. In herd 2 , goats with parity $>4$ were all recorded as parity $5+$. Parity data included in data analyses reflect parity at the time of enrollment (i.e., before dry-off).

\section{Sample Collection}

Two half-level consecutive milk samples were collected before dry-off, the first on the day of enrollment (14-21 d before planned dry-off) and the second 10 to $14 \mathrm{~d}$ later, and a further 2 consecutive half-level milk samples were collected after kidding, the first at $\leq 10$

Table 1. Demographic and management data for enrolled goats by herd

\begin{tabular}{llll}
\hline Variable & Herd 1 & Herd 2 & Herd 3 \\
\hline Herd size (female goats) & 1,200 & 150 & 42 \\
Number of goats enrolled & 115 & 136 & 37 \\
Type of production & Year round & Seasonal (Jan. to Nov.) & Seasonal (Feb. to Dec.) \\
Premilking teat disinfection & Iodine & Peroxide & Iodine \\
Postmilking teat disinfection & Iodine & Iodine & Iodine \\
Breed $^{1}$ & Saanen & Saanen and Alpine & Mancha \\
Parity $^{2}$ & $1(1-5)$ & $3(1-5)$ & $6(1-9)$ \\
DIM at dry-off $^{2}$ & $319(204-599)$ & $275(218-294)$ & $261(178-292)$ \\
Dry period duration, $^{2}$ d & $51(19-64)$ & $71(60-94)$ & $83.5(77-107)$ \\
Half-level pre-dry SCSS $^{2}$ & - & $7.7(2.4-9.6)$ & $7.0(3.2-9.6)$ \\
SRLV $^{3}$ seroprevalence, $\%$ (no./no.) & $44.3(51 / 115)$ & $91.2(124 / 136)$ & $0(0 / 37)$
\end{tabular}

${ }^{1}$ All herds were composed of mixed-breed goats; breeds indicated are the predominant phenotype in each herd.

${ }^{2}$ Median (range) among enrolled goats.

${ }^{3}$ Small ruminant lentivirus; prevalence among enrolled goats. 
DIM and the second 7 to $14 \mathrm{~d}$ later. All milk samples were collected before routine milking. Briefly, the udder was prepared according to the farm's premilking udder hygiene protocol by application of a teat disinfectant and drying of the teats with a single-use clean cloth or paper towel. Following routine premilking hygiene procedures, the teat ends were scrubbed with a cotton gauze soaked with $70 \%$ isopropyl alcohol. The first few streams of milk were discarded and milk from each udder half was aseptically collected into sterile plastic tubes for bacterial culture, transported on ice to the laboratory, and stored at $-20^{\circ} \mathrm{C}$ until analysis. For herds 2 and 3, half-level milk samples were collected into nonsterile vials containing a preservative tablet (2-bromo-2-nitropropane-1,3-diol; Broad Spectrum Microtabs II, D\&F Control Systems Inc., Dublin, CA) for determination of milk SCC and lactose concentration, refrigerated for $\leq 3 \mathrm{~d}$, and shipped overnight to a commercial laboratory for analysis (Mid-South Dairy Records, Springfield, MO).

At the time of study enrollment, whole-blood samples were collected into sterile evacuated glass vials by jugular venipuncture for determination of serum antibody titers against small ruminant lentivirus (SRLV) using a commercial test kit (see below), with the exception of 24 goats in herd 3 for which SRLV antibody titers had already been determined within 3 mo of study enrollment. Samples were allowed to clot; then, serum was separated by centrifugation $(1,700 \times g$ for $10 \mathrm{~min}$ at room temperature) and stored in plastic vials at $-20^{\circ} \mathrm{C}$ until analysis.

\section{SCC Enumeration and Lactose}

At the commercial laboratory, milk SCC were analyzed using an automated counter and milk lactose concentration was quantified by Fourier transform infrared absorption spectrometry (Bentley FTS/FCM, Bentley Instruments, Chaska, MN). Somatic cell score was computed using the equation $\mathrm{SCS}=\ln (\mathrm{SCC} / 100) / 0.6931$ +3 (Hogan et al., 1999).

\section{Milk Bacterial Culture}

Milk samples were thawed at room temperature $\left(\sim 22^{\circ} \mathrm{C}\right)$. Using a sterile cotton-tipped applicator swab, approximately $10 \mu \mathrm{L}$ of milk was plated onto Columbia blood agar (CBA; Remel, Lenexa, KS), and incubated at $37^{\circ} \mathrm{C}$ for $24 \mathrm{~h}$ and then at room temperature for a further $24 \mathrm{~h}$ (Roberson et al., 1994). Plates were assessed for growth at 24 and $48 \mathrm{~h}$. Samples that yielded $\geq 1$ colony on $\mathrm{CBA}$ were considered positive by culture. Colonies were presumptively identified based on morphology and hemolytic pattern. Mixed infections were defined as samples yielding 2 colonies of 2 different morphologies. Samples that yielded $\geq 3$ colonies of different morphology were defined as contaminated and excluded from subsequent analyses. Isolates from single and mixed infections were subcultured on CBA and stored in phosphate-buffered glycerol at $-80^{\circ} \mathrm{C}$ until further analysis.

Screening for Mycoplasma spp. was performed by creating a goat-level composite by mixing $20 \mu \mathrm{L}$ of milk from each udder-half sample together and plating onto half of a modified Hayflick Mycoplasma medium plate (Middleton et al., 2017) using a sterile cotton-tipped applicator swab. The milk was incubated at $35^{\circ} \mathrm{C}$ in $10 \% \mathrm{CO}_{2}$ for up to $10 \mathrm{~d}$, and plates were assessed for growth at 3,7 , and $10 \mathrm{~d}$. Samples that yielded $\geq 1$ colony of 1 to $2 \mathrm{~mm}$ in diameter with a "fried egg" appearance were defined as positive for Mycoplasma spp.

\section{Identification of Bacterial Isolates}

Initially, bacterial isolate identification was performed using MALDI-TOF MS (Bernier Gosselin et al., 2018). Colonies were obtained in pure culture and 2 to 3 colonies were used to spot onto the MALDI-TOF target plate in duplicate (Bruker Daltonics, Bremen, Germany). Each target was overlaid with $0.7 \mu \mathrm{L}$ of $70 \%$ formic acid, allowed to dry, and then overlaid with $1 \mu \mathrm{L}$ of matrix solution and allowed to dry. The plates were run on a MALDI-TOF mass spectrometer (Microflex LT/SH, Bruker Daltonics) and a score was assigned by the proprietary software to the spectrogram of each isolate, based on the similarity of the isolate's spectrum to the spectra of isolates in the proprietary library (Bruker Daltonics).

When a score $<2.0$ was assigned to a staphylococcal isolate, an extraction with ethanol and formic acid was performed by mixing colonies in $300 \mu \mathrm{L}$ of HPLC-grade water and $900 \mu \mathrm{L}$ of pure ethanol. After centrifugation at $12,100 \times g$ for $2 \mathrm{~min}$, the supernatant was discarded and the pellet was mixed with $5 \mu \mathrm{L}$ each of acetonitrile and $70 \%$ formic acid. The supernatant was spotted on the target plate, which was overlaid with matrix solution and the plate run as before.

A score of $\geq 2.0$ for at least one of the duplicates was considered adequate to name the isolate at the genus and species level. A score between 1.7 and 2.0 was considered adequate to name the isolate to the genus level (Tomazi et al., 2014). When the genus identification was not Staphylococcus, no further characterization of the isolate was performed; however, when an isolate was identified at the genus level as Staphylococcus but a species could not be assigned, further characterization of the isolate was performed using housekeeping gene sequence analysis (see below). A score of $<1.7$ meant 
that an isolate could not be identified at either the genus or species level, and no further characterization was performed. These isolates were considered unidentified.

If MALDI-TOF failed to yield a score of $\geq 2.0$ after formic acid extraction and the isolate was identified to the genus level as Staphylococcus, lysates of each isolate were prepared by mixing colonies from pure culture with $100 \mu \mathrm{L}$ of Tris-EDTA buffer. Then, PCR amplification and partial gene sequence analysis of rpoB, or tuf where rpoB was unsuccessful, were performed (Drancourt and Raoult, 2002; Hwang et al., 2011). The PCR products were purified using a PCR purification kit (PureLink, Invitrogen Life Technologies, Carlsbad, CA) and submitted to the University of Missouri DNA Core Facility for DNA sequencing. Gene sequences were compared with the GenBank database using the nucleotide-BLAST algorithm (https://blast.ncbi.nlm .nih.gov). Species identification was assigned if the isolate's rpoB sequence had $\geq 97 \%$ sequence similarity to a database sequence (Adkins et al., 2018) or if the tuf sequence had $\geq 98 \%$ similarity and $>0.8 \%$ separation from the next species (Hwang et al., 2011). If amplification was unsuccessful, DNA extraction was performed using a commercial kit (DNeasy kit, Qiagen, Valencia, CA) and amplification was repeated. If no species identification could be assigned following purification and gene sequence analysis, the isolate was considered unidentified.

\section{Strain-Level Identification of Staphylococcal Species}

When $>1$ sample from an udder half yielded the same staphylococcal species, the isolates from the first and last positive samples were compared at the strain level by PFGE, according to Middleton et al. (2002) with modification. Briefly, several colonies from a pure subculture of the stored isolate grown on CBA for 18 to $24 \mathrm{~h}$ at $37^{\circ} \mathrm{C}$ were suspended in buffer (Tris $0.1 \mathrm{M}$, EDTA $0.1 M, \mathrm{pH} 7.5$ ) to an optical density of 1.4 at $610 \mathrm{~nm}$. The resultant suspension was centrifuged, the supernatant discarded, and the pellet resuspended in $0.5 \mathrm{~mL}$ of $10 \mathrm{~m} M$ Tris, $0.1 M$ EDTA, and $10 \mathrm{mM}$ EGTA ( $\mathrm{pH} 8)$. The suspension was briefly incubated at $65^{\circ} \mathrm{C}$ and then mixed with $0.6 \mathrm{~mL}$ of $1 \%$ chromosomalgrade agarose to prepare plugs containing each isolate. Bacterial lysis and DNA digestion with SmaI were performed as previously described (Joo et al., 2001). The plugs were then placed in a $1 \%$ agarose gel immersed in $0.5 \%$ Tris-borate-EDTA buffer at $15^{\circ} \mathrm{C}$. The DNA fragments were separated by PFGE with a $120^{\circ}$ ramp angle and 5- to 50-s pulse time for $20 \mathrm{~h}$. The gel was stained with ethidium bromide and photographed by UV transillumination. The agar plugs for isolates to be compared were run side by side in the gel, the banding patterns (number and position of bands) were visually compared, and the isolates were considered the same strain if they had $100 \%$ similarity. When the first and last isolates from an udder half that was infected at multiple time points with the same species were a different strain type and the IMI was detected more than twice, the intervening samples were also strain-typed; otherwise, we assumed the intervening samples were of the same strain as the first and last isolates in the sample sequence.

\section{SRLV Status}

A commercial SRLV competitive ELISA (VMRD, Pullman, WA) was used according to the manufacturer's instructions to detect antibodies against SRLV. Serum samples were thawed at room temperature and run in duplicate. Optical density was measured by a microplate absorbance spectrophotometer at a wavelength of 630 $\mathrm{nm}$, and samples producing $\geq 35 \%$ inhibition compared with negative controls were considered positive. When the duplicates yielded discrepant results, another blood sample was collected at the time of milk sample 2 and testing was repeated. All of the cases for which testing was repeated showed agreement between duplicates.

\section{Statistical Analyses}

Definitions. Samples that were missing, contaminated, or resulted in the diagnosis of a non-staphylococcal or mixed IMI were classified as missing data (including SCC data) for the purposes of analysis. Pre-dry staphylococcal IMI status was defined using the 2 milk culture results interpreted in parallel, meaning that an udder half was considered infected if at least 1 of the 2 pre-dry samples was positive for a single staphylococcal species. If the 2 samples were positive but with different species or strain type within species between samples, the udder half was not included in the data analyses (i.e., these were considered as mixed IMI). If both predry samples were classified as missing data (see above), then the udder half was excluded from the study. Postkidding staphylococcal IMI status was similarly defined using the 2 post-kidding milk culture results.

Based on the species and strain-level identification data before and after the dry period and the IMI status definitions above, the following half-level IMI outcomes were defined. New IMI were halves with an IMI after kidding that were either culture-negative before dry-off or had an IMI with a different species or strain-type before dry-off. Cured IMI were halves with an IMI before dry-off that were culture-negative or culture-positive but with a different species or strain after kidding. Persistent IMI were halves with an IMI of the same species 
and strain before and after the dry period. Hence, some halves could experience both a cure of a pre-dry IMI and a new IMI with a different species or strain after kidding. Median and range for parity, DIM at dry-off, and dry-period duration for goats that kidded were computed for each herd.

For data analyses, pre-dry half-level milk quality parameters (MQP; SCS and lactose concentration) data from the second pre-dry sampling (closer to dry-off) were used. However, if the second sampling was classified as missing, data from the first sampling were used.

Estimation of IMI Cumulative Incidence and Cure Rate. Data from goats with complete culture and strain-typing data before and after kidding from all 3 herds were included. Descriptive statistics of halves with no growth before and after dry-off, new IMI, cured IMI, and persistent IMI, by species, were computed. For IMI incidence, because the halves with an IMI before dry-off could acquire an IMI caused by a different species or strain after kidding, both uninfected and infected halves before dry-off were considered at risk of new IMI. Incidence of new IMI over the dry period was calculated using a logistic regression model as a function of a baseline risk of new IMI, with herd- and goat-level random effects. Incidence was calculated for all species grouped together and for each species separately. For IMI cure, halves at risk of cure were halves with an IMI before dry-off. Cure was computed for all species grouped together, using a logistic regression model as a function of a baseline risk, with herd- and goat-level random effects.

Investigation of Factors Associated with IMI Dynamics Over the Dry Period. Only half-level pre-dry data for goats with complete culture data before and after kidding from herds 2 and 3 were included because no half-level SCS data were available for goats in herd 1. First, we built a generalized linear mixed regression model with a logit link to evaluate the association between half-level new IMI (all staphylococcal species grouped together) and half-level pre-dry SCS, after adjusting for confounding by pre-dry IMI status, SRLV status, and parity, and with a fixed herd effect and random goat effect. Both uninfected and infected halves before dry-off were considered at risk of a new IMI and therefore included in the model. The model was as follows:

$$
\begin{gathered}
\operatorname{logit}\left(\text { newIMI }_{\mathrm{ij}}\right)=\beta_{0}+\beta_{1} \mathrm{SCS}_{\mathrm{ij}}+\beta_{2} \mathrm{IMIPRE}_{\mathrm{ij}} \\
+\beta_{3} \mathrm{SRLV}_{\mathrm{j}}+\beta_{4} \text { Parity }_{\mathrm{j}}+\beta_{5} \operatorname{Herd}_{\mathrm{j}}
\end{gathered}
$$

where newIMI ${ }_{\mathrm{ij}}$ is the half-level new IMI status over the dry period $(0=$ no new IMI; $1=$ acquisition of a new IMI) of the ith udder half of the jth goat; $\beta_{0}$ is the in- tercept; $\beta_{1}$ is the coefficient for half-level pre-dry SCS; $\beta_{2}$ is the coefficient for pre-dry IMI status (IMIPRE; $0=$ no pre-dry IMI; $1=$ pre-dry IMI present); $\beta_{3}$ is the coefficient for SRLV status; $\beta_{4}$ is the coefficient for parity; and $\beta_{5}$ is the coefficient for herd.

Among halves with an IMI before dry-off, a second model was built to evaluate the association between IMI persistence and pre-dry half-level SCS, after adjusting for confounding by pre-dry half-level IMI species, SRLV status, and parity, and with a fixed herd effect and random goat effect. To improve model convergence, NAS species with $<10$ IMI before dry-off were grouped together in the category "other NAS." The model was as follows:

$$
\begin{gathered}
\operatorname{logit}\left(\text { Persist }_{\mathrm{ij}}\right)=\beta_{0}+\beta_{1} \text { SCS }_{\mathrm{ij}}+\beta_{3} \text { SRLV }_{\mathrm{j}} \\
+\beta_{4} \text { Parity }_{\mathrm{j}}+\beta_{5} \text { Herd }_{\mathrm{j}}+\beta_{6} \text { Species }_{\mathrm{ij}}
\end{gathered}
$$

where Persist ${ }_{\mathrm{ij}}$ is the half-level IMI persistence status $(0=$ nonpersistent IMI; $1=$ persistent IMI $)$ of the ith udder-half of the jth goat; $\beta_{6}$ is the coefficient for IMI species category; and other variables are as defined above.

Finally, among halves with an IMI before dry-off, a third model was built to evaluate the association between IMI persistence and pre-dry half-level IMI species, after adjusting for confounding by SRLV status and parity, and with a fixed herd effect and random goat effect. To improve convergence, $S$. aureus IMI were excluded $(\mathrm{n}=2)$. The model was as follows:

$$
\begin{gathered}
\operatorname{logit}\left(\text { Persist }_{\mathrm{ij}}\right)=\beta_{0}+\beta_{6} \text { Species }_{\mathrm{ij}}+\beta_{3} \text { SRLV }_{\mathrm{j}} \\
+\beta_{4} \text { Parity }_{\mathrm{j}}+\beta_{5} \text { Herd }_{\mathrm{j}},
\end{gathered}
$$

with variables defined as above.

MQP Thresholds to Predict IMI Dynamics. Mixed logistic regression models were built to evaluate the association between different half-level outcomes (described below) and MQP. If the final model identified significant interactions between MQP and another independent variable (pre-dry half-level IMI species, SRLV status, parity), different MQP thresholds were calculated for different classes of this variable. If the model showed an association between MQP and the outcome of interest with a $P$-value $>0.10$, then computation of a threshold for predicting the outcome was not attempted. The optimal threshold for MQP to detect the outcome of interest was calculated using receiver operating characteristic analysis. The Youden index was computed from the sensitivity and specificity of a range of probabilities, and the probability with the highest Youden index value was used to back-calculate the MQP threshold. 
Half-level pre-dry data could be useful to detect halves of goats that may benefit from intervention such as dry-off therapy or culling. In situations when a pre-dry culture is positive for a staphylococcal species and MQP data are known, the optimal threshold for half-level MQP to predict persistence of pre-dry IMI was calculated using receiver operating characteristic analysis as described above.

Additionally, using data from all halves, a similar model was evaluated to determine the optimal threshold for half-level MQP to detect pre-dry IMI that would persist, irrespective of culture data. Therefore, this threshold could be used as a screening tool to select halves for bacterial culture.

Model Building. For all models described, significance was set at $P<0.05$. Unconditional relationships between the independent variables and the outcome variables were first evaluated. Linearity of the relationship between outcome variables and continuous independent variables was evaluated with polynomial square and cubic terms, and significant polynomial terms were included in the models. Interactions between IMI status or species and SRLV status or parity were assessed. Normality and homoscedasticity of residuals were visually assessed. Generalized linear mixed regression analyses were conducted using the SAS 9.4 GLIMMIX procedure (SAS Institute Inc., Cary, NC). All analyses were performed using SAS 9.4 (SAS Institute Inc.).

\section{RESULTS}

Before dry-off, 288 goats were initially enrolled. Herd 1 was sold and depopulated during the course of the study, resulting in 72 goats not having kidded before being lost to follow-up. Additionally, 13 goats from herd 2 and 3 goats from herd 3 did not kid before the end of the study. Therefore, 200 goats completed the study, including 43 goats from herd 1, 123 from herd 2, and 34 from herd 3. The data for parity, DIM at dry-off, duration of the dry period, half-level SCS before dry-off, and SRLV status for the goats enrolled are presented in Table 1.

No Mycoplasma spp. were detected by culture (detection threshold $=25 \mathrm{cfu} / \mathrm{mL}$ ). After exclusion of samples classified as missing, 380 halves from 198 goats had complete culture, staphylococcal speciation, and strain-typing data; 240 halves had no growth before and after dry-off. Among the remaining 140 halves, the number of IMI outcomes by each staphylococcal species is presented in Table 2. Ten halves had both a cured IMI and a new IMI. The prevalence of IMI was $26.3 \%$ (100/380) before dry-off and $25.8 \%$ (98/380) after kidding. The incidence $(95 \% \mathrm{CI})$ of new IMI at the half level was $13.2 \%$ (9.8-17.4); species-specific incidences are presented in Table 2. The proportion (95\% CI) of pre-dry IMI that were cured was $52.0 \%$ (42.1-61.7).

\section{Investigation of Factors Associated with IMI Dynamics During the Dry Period}

After exclusion of 81 halves from herd 1 (no SCS data), the model investigating the risk factors for new IMI included data from 299 halves. When evaluating unconditional association, new IMI status was not associated with SRLV status. In the final SCS model, after adjusting for confounding by pre-dry IMI status,

Table 2. Number of half-level IMI events (new IMI, cured IMI, and persistent IMI) among 140 udder halves with a staphylococcal IMI and species and strain-type data, and incidence and proportion of cured and persistent IMI

\begin{tabular}{|c|c|c|c|c|c|}
\hline Item & $\begin{array}{l}\text { New } \\
\text { IMI }\end{array}$ & $\begin{array}{l}\text { Incidence, } \\
\%(95 \% \text { CI })\end{array}$ & $\begin{array}{c}\text { Cured IMI, } \\
\text { no. }(\%)\end{array}$ & $\begin{array}{c}\text { Persistent IMI, } \\
\text { no. }(\%)\end{array}$ & Total $^{1}$ \\
\hline Total IMI events & 50 & $13.2(9.8-17.4)$ & $52(52)$ & $48(48)$ & 150 \\
\hline \multicolumn{6}{|l|}{ Staphylococcus spp. } \\
\hline S. aureus & 2 & $0.5(0.1-2.1)$ & $1(50)$ & $1(50)$ & 4 \\
\hline S. caprae & 24 & $6.3(4.1-9.6)$ & $9(39.1)$ & $14(60.9)$ & 47 \\
\hline S. simulans & 12 & $3.2(1.7-5.7)$ & $5(25)$ & $15(75)$ & 32 \\
\hline S. arlettae & 0 & - & $16(100)$ & 0 & 16 \\
\hline S. epidermidis & 0 & - & $5(38.5)$ & $8(61.5)$ & 13 \\
\hline S. xylosus & 6 & $1.6(0.7-3.4)$ & $2(33.3)$ & $4(66.7)$ & 12 \\
\hline S. equorum & 2 & $0.5(0.1-2.1)$ & $6(100)$ & 0 & 8 \\
\hline S. lentus & 2 & $0.5(0.1-2.1)$ & $5(100)$ & 0 & 7 \\
\hline S. chromogenes & 1 & $0.3(0.04-1.8)$ & $1(33.3)$ & $2(66.7)$ & 4 \\
\hline S. warneri & 1 & $0.3(0.04-1.8)$ & 0 & $2(100)$ & 3 \\
\hline S. cohnii & 0 & - & $1(50)$ & $1(50)$ & 2 \\
\hline S. auricularis & 0 & - & $1(100)$ & 0 & 1 \\
\hline S. lugdunensis & 0 & - & 0 & $1(100)$ & 1 \\
\hline
\end{tabular}

${ }^{1}$ Total of new IMI, cured IMI, and persistent IMI for all halves and for each species. Ten halves had a pre-dry period IMI that was cured and followed by a new IMI by a different species or strain after kidding, and were therefore counted twice. 
SRLV status, and parity, new IMI status was not associated with pre-dry SCS $(P=0.64)$. An increase in the pre-dry SCS of 1.0 was associated with 1.1 (95\% CI: 0.79-1.5) times greater odds of new IMI.

Among 100 halves with IMI before dry-off (Table 2), 37 halves from herd 1 were excluded (no SCS data). Therefore, the models investigating the risk factors for IMI persistence included data from 63 halves. Staphylococcal species were categorized as $S$. aureus, $S$. caprae, S. simulans, and "other NAS" (i.e., S. arlettae, $S$. chromogenes, S. cohnii, S. epidermidis, S. equorum, $S$. lentus, S. warneri, and S. xylosus). When evaluating unconditional associations, IMI persistence was not associated with SRLV status. In the final pre-dry SCS model, after adjusting for confounding by pre-dry IMI species, SRLV status, and parity, persistence of IMI was not associated with pre-dry $\operatorname{SCS}(P=0.10)$. An increase in the pre-dry SCS of 1.0 was associated with 1.5 (95\% CI: 0.91-2.6) times greater odds of IMI persistence.

In the final IMI species model, after adjusting for confounding by SRLV status and parity, persistence of IMI was not associated with pre-dry IMI species $(P=$ 0.13). When compared with $S$. simulans IMI, S. caprae IMI and "other NAS" IMI had 0.86 (95\% CI: 0.14-5.3) times and 0.17 (95\% CI: $0.02-1.2)$ times lower odds of persistence, respectively.

\section{MQP Thresholds to Predict IMI Dynamics}

No significant interaction between MQP and other variables was identified in any of the models evaluated. Associations between lactose concentration and the various outcomes all yielded $P$-values $>0.10$ in the models evaluated. Pre-dry SCS, however, yielded an association with IMI persistence with a $P$-value $\leq 0.10$, and was therefore investigated for predicting IMI persistence. For halves with known pre-dry staphylococcal IMI, the model with pre-dry SCS yielded an area under the curve of 0.66 (95\% CI: 0.52-0.79; Figure 1a), and the optimal pre-dry SCS threshold to predict IMI persistence was 8.6 (corresponding to a SCC of $4,717 \times 10^{3}$ cells $\left./ \mathrm{mL}\right)$, with sensitivity $(95 \% \mathrm{CI})$ and specificity (95\% CI) of $53.1 \%(29.1-65.3)$ and $80.6 \%$ (62.5-92.6), respectively. Among all halves (regardless of culture data), the model yielded an area under the curve of 0.64 (95\% CI: 0.54-0.73; Figure 1b), and the optimal pre-dry SCS threshold to predict the presence of a staphylococcal IMI that would persist was 8.7 (corresponding to a $\mathrm{SCC}$ of $5,235 \times 10^{3}$ cells $/ \mathrm{mL}$ ), with sensitivity (95\% CI) and specificity (95\% CI) of $50 \%$ (31.9-68.1) and $73.8 \%$ (68.1-79.0), respectively.

\section{DISCUSSION}

The first objective of this study was to describe the dynamics of staphylococcal IMI around the dry
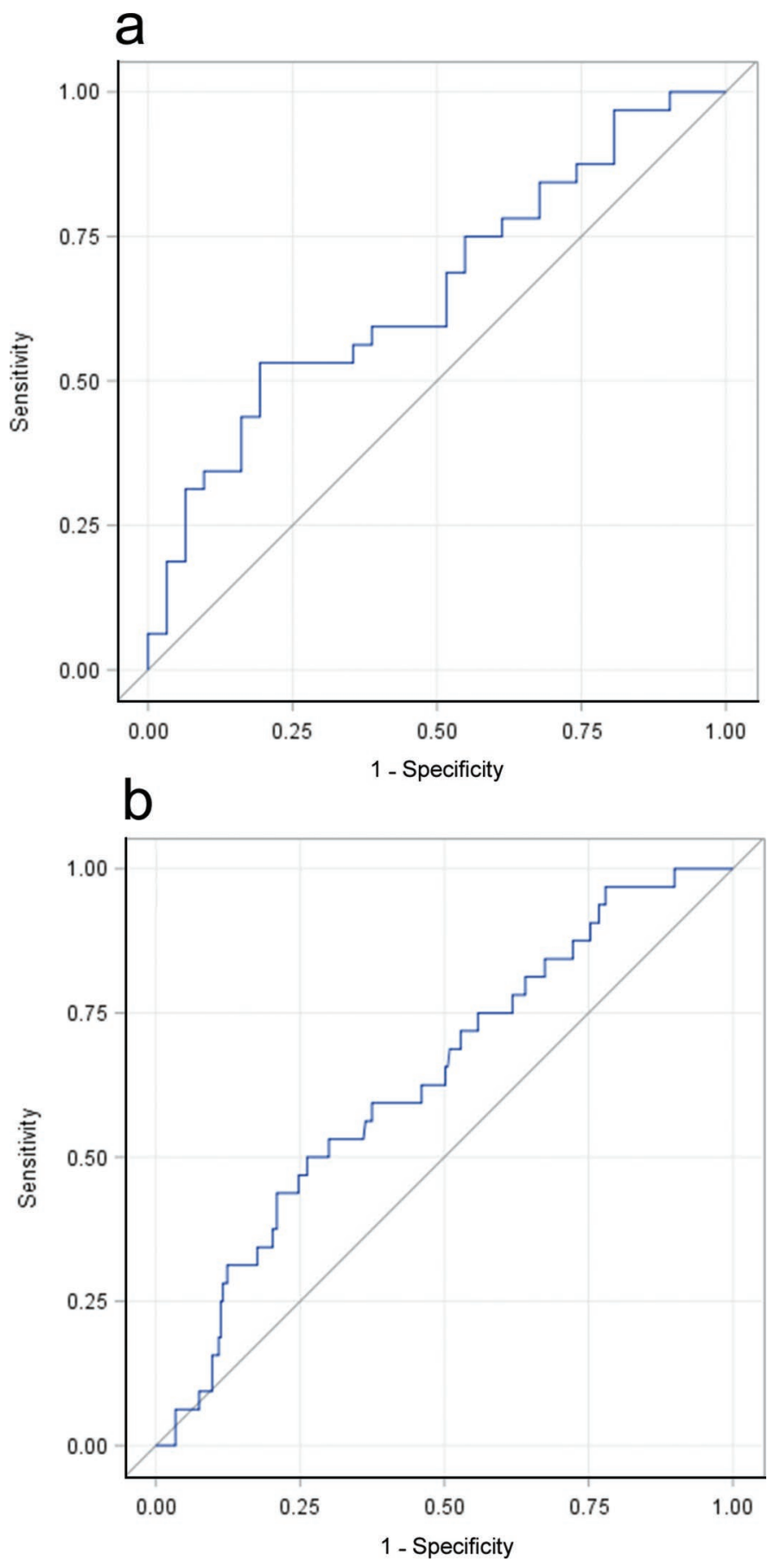

Figure 1. Receiver operating characteristic curves of half-level predry SCS to predict pre-dry IMI that would persist into the next lactation, among halves with known positive IMI status before dry-off (a) and among all halves regardless of pre-dry IMI status (b). 
period. The prevalences before dry-off and after kidding were similar to one another $(\sim 25 \%)$ and higher than prevalences in early lactation reported by others (McDougall et al., 2010, 2014). Our definition of IMI status was based on parallel interpretation of 2 samples for each period (pre-dry and post-kidding) to increase the sensitivity of culture to detect an IMI at each period, which could explain in part the higher observed prevalence. New staphylococcal IMI rates during the dry period were similar to those previously reported (Fox et al., 1992; Leitner et al., 2007). An unexpected observation was the high proportion of new IMI that were caused by S. caprae (48\%) compared with other common staphylococcal species. For each of the 3 study herds, S. caprae caused 100, 56, and $8 \%$ of new IMI, respectively. These results contrast with those reported by Leitner et al. (2007), where $59.1 \%$ of new IMI were caused by $S$. simulans compared with $S$. epidermidis $(4.5 \%)$ and $S$. caprae $(0 \%)$. Within a given species, pathogenicity may vary between strains (Vanderhaeghen et al., 2014); therefore, different strains may result in differences in incidence (or cure) rates between herds and between studies. Nevertheless, the results of the present study suggest that the dry period may be an important time in the production cycle for acquisition of $S$. caprae IMI in some herds, and therefore represents a period with potential for control measures to reduce the incidence of IMI. Although it is unknown whether these new IMI persisted beyond the first month in lactation, a previous study found that IMI detected in early lactation were more likely to persist than IMI detected after 10 DIM (Bernier Gosselin et al., 2019).

The IMI cure rates during the dry period were higher than in previous reports, which reported cure rates of $19.7 \%$ for CNS IMI in untreated halves and ranged from 32.8 to $82.0 \%$ in halves that received intramammary antibiotic therapy at dry-off (Fox et al., 1992; Poutrel et al., 1997; Leitner et al., 2007). In the present study, $S$. arlettae was common among pre-dry staphylococcal IMI, but none of these IMI persisted, which is similar to previous observations during lactation (Bernier Gosselin et al., 2019).

Goat-level factors that have been reported or hypothesized to be associated with increased risk of IMI (regardless of phase of the lactation cycle) include breed, parity, duration of the dry period, SRLV infection, level of milk production, and position of the udder (East et al., 1987; Contreras et al., 1999; Moroni et al., 2005a,b; Koop et al., 2013). Additionally, an association between SCS and IMI persistence during lactation was previously reported (Moroni et al., 2005a), which could reflect that pathogenic staphylococcal strains able to persist in the mammary gland also induce a higher SCS response. In the present study, pre-dry SCS was investigated as a potential risk factor for new IMI and for IMI persistence but was not significantly associated with any of these outcomes. Finally, IMI species was not associated with odds of persistence; however, because of the small number of isolates within each species, most species were grouped together, which may have leveled out species-specific differences.

Regardless of whether culture data were included in the model, optimal pre-dry SCS thresholds lacked sensitivity and had only moderate specificity for detecting IMI that would persist over the dry period into the subsequent lactation. The model did not differentiate between uninfected halves and those with an IMI that would be eliminated, for which a different threshold could be computed. With a similar aim of detecting goats that may benefit from dry-off therapy, a different approach was reported by Baudry et al. (1999), which used multiple monthly composite SCC above a given threshold during the entire lactation instead of a single pre-dry measurement as used in the present study. In that report, thresholds were suggested for detection of $S$. aureus IMI and CNS IMI, although, for the latter, a threshold was only determined for primiparous goats and could not be established for multiparous goats (Baudry et al., 1999). Data from the present study suggest that many NAS species are associated with persistence of IMI over the dry period and into the next lactation. The possibility of detecting such IMI using combinations of SCS and staphylococcal species isolated from milk cultures or data from multiple testdays warrants further investigation.

Seropositivity to SRLV has been associated with higher SCC in goats free of bacterial IMI; it is reported to affect the SCC response to bacterial IMI and is hypothesized, therefore, to dampen the immune response of the mammary gland (Sánchez et al., 2001). In the present study, SRLV status was not associated with incidence of IMI or IMI persistence during the dry period. The specific cell populations and mechanisms involved in the potential interaction and confounding of SRLV on the association between bacterial IMI and SCC need further investigation. Because SRLV infection can initially be latent before causing progressive inflammation, it might have a wide range of effects on the mammary gland SCC and response to IMI, and seropositivity may or may not accurately correlate with the continuum of the disease. Nevertheless, at the herd level, control of SRLV seroprevalence could be beneficial in the control of IMI incidence. Furthermore, because of its apparent effect on the risk of IMI and on SCC, SRLV status should be considered a confounding factor in studies investigating the association between bacterial IMI and SCC. 
In conclusion, a significant proportion of pre-dry NAS IMI persisted into the next lactation, and this proportion varied between species. Further investigation is warranted regarding species-specific spontaneous cure rate, the effects of interventional strategies at dry-off on species-specific incidence and cure rates, and on the role of SRLV infection in IMI dynamics.

\section{ACKNOWLEDGMENTS}

The authors thank Samantha Castillo, Katy Kaufman, Elizabeth-Blair McKinnon, Luiza Placheta, and Jacob Wilshusen (University of Missouri, Columbia) for their assistance in sample collection; the farm personnel for their collaboration; and Julie Holle, Kaitlyn Webb, Mark Foecking, LaToyia Sly, and Amber Mann (University of Missouri, Columbia) for technical assistance. This study was supported by the USDA National Institute of Food and Agriculture (Washington, DC; project no. AH2631845, 2017-2018).

\section{REFERENCES}

Adkins, P. R. F., S. Dufour, J. N. Spain, M. J. Calcutt, T. J. Reilly, G. C. Stewart, and J. R. Middleton. 2018. Molecular characterization of non-aureus Staphylococcus spp. from heifer intramammary infections and body sites. J. Dairy Sci. 101:5388-5403.

Bastan, A., S. Salar, D. Baki Acar, M. A. Demirel, M. Cengyz, I. Darbaz, and G. Bulut. 2015. The effects of dry-off therapy on milk somatic cell count in Saanen goats. Turk. J. Vet. Anim. Sci. 39:550-555.

Baudry, C., P. Mercier, M. P. Mallereau, and D. Lenfant. 1999. Utilisation des numérations cellulaires individuelles pour la detection des infections mammaires subcliniques de la chèvre: définition de seuils. Pages 119-123 in Proc. 6th Int. Symp. Milking Small Rumin., Athens, Greece. EAAP Publ. No. 95. Wageningen Pers, Wageningen, the Netherlands.

Bernier Gosselin, V., S. Dufour, P. R. F. Adkins, S. Poock, P. Pithua, and J. R. Middleton. 2019. Longitudinal microbiological evaluation of subclinical non-aureus staphylococcal intramammary infections in a lentivirus-infected dairy goat herd. Vet. Microbiol. 230:156-163.

Bernier Gosselin, V., J. Lovstad, S. Dufour, P. R. F. Adkins, and J. R. Middleton. 2018. Use of MALDI-TOF to characterize staphylococcal intramammary infections in dairy goats. J. Dairy Sci. 101:6262-6270.

Contreras, A., J. C. Corrales, A. Sanchez, and D. Sierra. 1997. Persistence of subclinical intramammary pathogens in goats throughout lactation. J. Dairy Sci. 80:2815-2819.

Contreras, A., J. C. Corrales, D. Sierra, and J. Marco. 1995. Prevalence and aetiology of non-clinical intramammary infection in Murciano-Granadina goats. Small Rumin. Res. 17:71-78.

Contreras, A., M. J. Paape, and R. H. Miller. 1999. Prevalence of subclinical intramammary infection caused by Staphylococcus epidermidis in a commercial dairy goat herd. Small Rumin. Res. 31:203-208.

Drancourt, M., and D. Raoult. 2002. rpoB gene sequence-based identification of Staphylococcus species. J. Clin. Microbiol. 40:1333-1338.

East, N. E., E. F. Birnie, and T. B. Farver. 1987. Risk factors associated with mastitis in dairy goats. Am. J. Vet. Res. 48:776-779.

Fox, L. K., D. D. Hancock, and S. D. Horner. 1992. Selective intramammary antibiotic therapy during the nonlactating period in goats. Small Rumin. Res. 9:313-318.
Hogan, S. J., R. N. González, R. J. Harmon, S. C. Nickerson, S. G. Oliver, J. W. Pankey, and K. L. Smith, ed. 1999. Laboratory Handbook on Bovine Mastitis. Rev. ed. National Mastitis Council, Madison, WI.

Hwang, S. M., M. S. Kim, K. U. Park, J. Song, and E. C. Kim. 2011. tuf gene sequence analysis has greater discriminatory power than $16 \mathrm{~S}$ rRNA sequence analysis in identification of clinical isolates of coagulase-negative staphylococci. J. Clin. Microbiol. 49:4142-4149.

Joo, Y. S., L. K. Fox, W. C. Davis, G. A. Bohach, and Y. H. Park. 2001. Staphylococcus aureus associated with mammary glands of cows: Genotyping to distinguish different strains among herds. Vet. Microbiol. 80:131-138.

Koop, G., C. A. Collar, N. Toft, M. Nielen, T. van Werven, D. Bacon, and I. A. Gardner. 2013. Risk factors for subclinical intramammary infection in dairy goats in two longitudinal field studies evaluated by Bayesian logistic regression. Prev. Vet. Med. 108:304-312.

Leitner, G., U. Merin, O. Krifucks, S. Blum, A. L. Rivas, and N. Silanikove. 2012. Effects of intra-mammary bacterial infection with coagulase negative staphylococci and stage of lactation on shedding of epithelial cells and infiltration of leukocytes into milk: comparison among cows, goats and sheep. Vet. Immunol. Immunopathol. 147:202-210.

Leitner, G., U. Merin, Y. Lavi, A. Egber, and N. Silanikove. 2007. Aetiology of intramammary infection and its effect on milk composition in goat flocks. J. Dairy Res. 74:186-193.

McDougall, S., D. Malcolm, and C. Prosser. 2014. Prevalence and incidence of intramammary infections in lactating dairy goats. N. Z. Vet. J. 62:136-145.

McDougall, S., K. Supré, S. De Vliegher, F. Haesebrouck, H. Hussein, L. Clausen, and C. Prosser. 2010. Diagnosis and treatment of subclinical mastitis in early lactation in dairy goats. J. Dairy Sci. 93:4710-4721

Middleton, J. R., L. K. Fox, J. M. Gay, J. W. Tyler, and T. E. Besser. 2002. Influence of Staphylococcus aureus strain-type on mammary quarter milk somatic cell count and N-acetyl-beta-D-glucosaminidase activity in cattle from eight dairies. J. Dairy Sci. 85:11331140 .

Middleton, J. R., L. K. Fox, G. Pighetti, and C. Petersson-Wolfe, ed. 2017. Laboratory Handbook on Bovine Mastitis. 3rd ed. National Mastitis Council, New Prague, MN.

Moroni, P., G. Pisoni, M. Antonini, G. Ruffo, S. Carli, G. Varisco, and P. Boettcher. 2005a. Subclinical mastitis and antimicrobial susceptibility of Staphylococcus caprae and Staphylococcus epidermidis isolated from two Italian goat herds. J. Dairy Sci. 88:1694-1704.

Moroni, P., G. Pisoni, G. Ruffo, and P. J. Boettcher. 2005b. Risk factors for intramammary infections and relationship with somaticcell counts in Italian dairy goats. Prev. Vet. Med. 69:163-173.

Poutrel, B. 1984. Udder infection of goats by coagulase-negative staphylococci. Vet. Microbiol. 9:131-137.

Poutrel, B., R. de Cremoux, M. Ducelliez, and D. Verneau. 1997. Control of intramammary infections in goats: impact on somatic cell counts. J. Anim. Sci. 75:566-570.

Roberson, J. R., L. K. Fox, D. D. Hancock, J. M. Gay, and T. E. Besser. 1994. Ecology of Staphylococcus aureus isolated from various sites on dairy farms. J. Dairy Sci. 77:3354-3364.

Sánchez, A., A. Contreras, J. C. Corrales, and J. C. Marco. 2001. Relationships between infection with caprine arthritis encephalitis virus, intramammary bacterial infection and somatic cell counts in dairy goats. Vet. Rec. 148:711-714.

Tomazi, T., J. L. Goncalves, J. R. Barreiro, P. A. de Campos Braga, L. F. Prada e Silva, M. N. Eberlin, and M. V. dos Santos. 2014. Identification of coagulase-negative staphylococci from bovine intramammary infection by matrix-assisted laser desorption ionizationtime of flight mass spectrometry. J. Clin. Microbiol. 52:1658-1663.

Vanderhaeghen, W., S. Piepers, F. Leroy, E. Van Coillie, F. Haesebrouck, and S. De Vliegher. 2014. Effect, persistence, and virulence of coagulase-negative Staphylococcus species associated with ruminant udder health. J. Dairy Sci. 97:5275-5293.

White, E. C., and L. S. Hinckley. 1999. Prevalence of mastitis pathogens in goat milk. Small Rumin. Res. 33:117-121. 